\title{
CONCEPTS OF SIMULATION MODEL SIZE AND COMPLEXITY
}

\author{
Ahmed, R. ; Shah, M.** \& Umar, M. ${ }^{* * *}$ \\ ${ }^{*}$ Lahore School of Economics, Burki Road, Lahore, Pakistan \\ ${ }^{* *}$ Lancashire Business School, University of Central Lancashire, UK \\ *** Department of Global Value Chains, Lincoln University, Christchurch, New Zealand \\ E-Mail: rizwan.ahmed@lahoreschool.edu.pk,mhshah@uclan.ac.uk, \\ Muhammad.umar@lincolnuni.ac.nz
}

\begin{abstract}
Simulation modelling is the business of reducing complexity of the real world. Keep your models small and simple, is the basic heuristic found in simulation \& modelling literature; yet the modellers are developing models of increasing size and complexity. Simulation model size and complexity is generally a less debated and poorly understood area. Despite the importance of model simplification and its relation with size and complexity; only a handful of papers, addressing the size and complexity of simulation models, can be found. This paper reports on general concepts of simulation model size and complexity held by expert modellers and proposes a framework to understand the origins of simulation model size and complexity. Size and complexity are two most important contextual factors that may affect simulation practice. Increased understanding of concepts of simulation model size and complexity held by the modellers will potentially underpin simulation methodology research and help us in addressing the issues raised by increasing size and complexity of simulation models.

(Received in February 2015, accepted in November 2015. This paper was with the authors 1 month for 1 revision.)
\end{abstract}

Key Words: Simulation Model Size, Model Complexity, Context, Methodology

\section{INTRODUCTION}

Simulation modelling is the business of reducing complexity of the real world. Simulation modellers study complex problems, build conceptual models, and transform those conceptual models into computer models to represent and study the real world complex phenomenon in a simple manner. Yet the modellers, whose primary objective is to reduce the complexity of the real world, are developing models of increasing size and complexity [1-2]. Simulation model size and complexity continues to grow with the growing computing power and sophistication of simulation tools. Researchers have highlighted the areas in which issues may arise due to growing size and complexity; such as model conceptualization, validation and verification, correctness, accuracy, ease of use, understanding, maintenance, reusability, performance and cost [1-5]. However, there seems to be little interest in defining simulation model size and complexity and understanding the factors responsible for the increasing size and complexity. The question is seldom asked whether simulation model size and complexity has any effect on the cost, time, and the way people go about developing simulation models. How could simulation model size and complexity be measured? Is it even important for practitioners and researchers to measure simulation model size and complexity? And would it help if we are able to appreciate the factors responsible for increased size and complexity?

The objective of this paper is to report on the general concepts of simulation model size and complexity held by 20 expert simulation modellers and to understand the origins of simulation model size and complexity. The results are part of an in-depth study exploring the contexts and practices of simulation modellers, partly reported in an earlier paper in IJSIMM [6]. The contribution of this paper lies in underpinning the understanding of simulation model size and complexity and suggesting a framework to understand the origins of size and 
complexity. We believe that an appreciation of the origins of size and complexity would help reducing model size and complexity and thus producing better and credible models.

In the next section we discuss our literature findings on simulation model size and complexity. After that we briefly outline our research methodology and then present our findings from semi-structured interviews. Finally we discuss and relate our findings with the literature; and conclude the discussion along with pointers to future directions.

\section{SIMULATION MODEL COMPLEXITY AND SIZE IN THE LITERATURE}

Keep your model small and simple, is a general heuristic found in simulation literature and curriculum [1, 7-10], yet the modellers are developing models of increasing size and complexity [1-2]. Several factors may contribute towards making a simulation model large and complex depending on the context. In some simulation interventions, termed by Robinson (2002) as simulation as software engineering, the simulation models produced are usually large and highly complex. On other occasions, when simulation is being conducted in the mode of organisational change or facilitation [7], the complexity and size increases in the model partly due to lack of experience and 'include all' syndrome [2]. Degree of sophistication and capability of simulation software may have an effect on simulation model size and complexity [1,6]. Chwif et al. [2] say that higher complexity and bigger size not only affect the performance of a simulation model but also other aspects such as required resources and development time. Chwif et al. [2] say, "Size and complexity of models is growing more and more, forcing modellers to face some problems that they were not accustomed to".

Simulation size and complexity appears to be a low priority and less attended issue amongst simulation community yet in the words of Henrikson [1], complexity is the enemy\# 1 . Arthur et al. [5] note that simulation model size and complexity continues to grow. This growing phenomenon brings some new issues along with it; as Henrikson [1] says that model correctness, ease of use, performance and cost are adversely affected by complexity of the simulation model. Arthur et al. [5] believe that verification and validation are becoming difficult for large and increasingly complex models and discuss possible avenues to solve this problem. They claim that as the size and complexity of a simulation model increases the cost associated with field testing the models increases. Thus on many occasion modellers tend to avoid field testing and rely only on synthetic data. Page et al. [11] raise some very important questions which have arisen with increasing scale of simulation models;

- How should a huge model be expressed?

- How should a huge model be validated?

- How to solve a huge model?

- How should the output of a huge model be traced and understood?

Moreover, Astrup et al. [4] suggest that the predictive ability of a simulation model may be affected with the size and complexity of a model. In an attempt to choose the best model for studying forest growth, they found that the most complex models had the poorest predictive ability where as models with intermediate complexity had better predictive ability. Astrup et al. [4] view complexity on two different levels; first, at a higher level of abstraction where the objective is to find out if model structure corresponds to model objectives; second, at a lower level of abstraction as in appropriateness of individual functions applied within the model to the available data.

Jacobson and Yucesan [12] believe that if metrics of simulation complexity are established they can be a very useful attribute in a priori evaluation of proposed simulation studies that are required to be completed in a limited budget. Similarly Brooks and Tobias [13] recommend that establishing complexity metrics may significantly help in choosing the 
best model out of various alternative models. Jacobson and Yucesan [12] propose graph theoretic approach to measure the complexity of simulation models.

Brooks and Tobias [13] discuss simulation model size and complexity from a theoretical and conceptual standpoint; however, it is hard to find a generally agreed definition and metrics of size and complexity of simulation models in the literature [2]. Since perception of size and complexity may vary under varying context and modellers' own experience and background, it is difficult to establish metrics for size and complexity. Simulation literature does not report any such study where modellers' concepts of size and complexity have been explored. Exploring the concepts of simulation model size and complexity may help us move toward establishing some workable definition and metrics for size and complexity.

Given the increase in size and complexity of models with the availability of increased computing power, simulation researchers and practitioners need to develop an understanding of simulation size and complexity. Size and complexity are two of the most important contextual factors that may affect simulation practice. Increased understanding of concepts of simulation model size and complexity will potentially underpin simulation methodology research and help practitioners develop better model and be cost effective. This research contributes towards enhanced understanding of the concepts of simulation model size and complexity held by the participants of our study.

\section{METHODOLOGY}

This research was conducted using semi-structured interviews. The interview questions focused on 8 main areas of interest to explore the modelling context and practices of the modellers; size and complexity was one of eight areas. An interview questions pool consisting of open ended questions along an interview script document was prepared, which was used during the interview. Grounded theory approach was followed for coding interview transcripts and subsequent data analysis. In grounded theory the researcher looks for themes or concepts with an open mind and every new theme identified is noted, defined, and labelled against the data. As such an inductive approach is used in which the data categories emerge in an evolutionary manner from the data.

We also conducted an extensive pilot study consisting of two phases with an objective to evaluate the interviewing instrument. First phase aimed to pre-test the validity of questions to be asked in the interview. The initial draft of the questions pool was then improved on the basis of feedback received by the pre-testing participants. In the second phase, pilot interviews were conducted with four other participants, which helped us in assessing the appropriateness, structure, and flow of the interview. This also provided us practice for the main set of interviews; and helped us determine appropriate duration of the interviews and evaluate the audio recording equipment.

Twenty participants in total from USA, UK, Germany, Spain and South Africa took part in this study; out of which 14 participants held $\mathrm{PhD}, 3$ participants held Master, and 3 participants hold Bachelor degree qualification. This suggests that the participants in this study are highly educated and most of them had some modelling education as part of their professional or research degrees. The average experience of the participants in simulation is 8.5 years.

As stated earlier that the results in this paper are part of an in-depth study exploring the contexts and practices of simulation modellers; which has been partly reported in an earlier paper in IJSIMM by Ahmed \& Shah [6]. Readers may refer to this paper for further details on our methodology, participants selection and data analysis. 


\section{RESULTS}

This section reports the results on concepts simulation model size and complexity. When the participants were asked about the size and complexity of their models, most of them did not have a clear idea how to answer it. It appeared that most of them never explicitly thought about the size and complexity of their models. Typical reaction by the participant has been summarized in the Exhibit I below. However, with follow up questions, the participants opened up and expressed their views on simulation model size and complexity. It appeared that most participants relate size with complexity and vice versa. Following section present the results on participant's views on size and complexity of their simulation models.

Exhibit I: Participants reaction when asked about size and complexity of their models.

- What do you mean by size?

- It is difficult to decide what you mean by size

- Depends how you define size and complexity

- It is hard to say

- I don't know

- I haven't thought about it

- I measure in dollars

- On what metric?

- It's very difficult to know again what you mean by complexity

- How do you quantify size?

- What do you mean by big? Is it file size or...?

- Yeah... so it is big... but if you ask me how big then you have to tell me how you measure the size...

\subsection{Size of simulation models}

Our participants develop simulation models of varying size and complexity, however, the concept of size and complexity held by each participant is different. Table I shows that 3 participants perceive their models to be small, 14 participants consider their models medium in size, and 3 participants think that they develop large models. This suggests that majority of the participants mostly develop simulation models of small and/or medium complexity.

We found no agreement or a common metric for simulation model size. Table I shows various potential metrics indicated by the participants to measure simulation model size. Most participants like to perceive size of the model in terms of problem magnitude, for example, number of entities, elements, activities or process steps. Some participants would like to measure model size in terms of the time it takes to develop the model as shown by the transcript excerpt of S12 in Exhibit II. Participants who use Witness or Extend also tend to measure size in terms of the number of 'blocks' in the model. Some participants also think that the number of variables can be a measure of size. Few participants think that lines of code will be a good measure for the size of a simulation model. Amount of input data is another potential metric to measure model size as S12, S14 and S17 think that more the input data the bigger the model is going to be in terms of execution and data processing.

S1 also suggests model size cannot be measured independent of simulation software. Programming language or simulation software used is an important factor that may affect model size. A model developed in Java or Visual Basic may appear very big in terms of lines of code, however, the same model may appear very small in Witness or Extend due to the direct support in terms of model constructs and visual components. 
Table I: Size of the models developed by the participants, and possible measures of size.

\begin{tabular}{|c|c|c|c|c|c|c|c|}
\hline Participants & Size & Time & $\mathrm{NOV}$ & NOEAP & $N O B$ & $L O C$ & $A M I D$ \\
\hline S1 & Small & & $\mathrm{Y}$ & & $\mathrm{Y}$ & & \\
\hline $\mathbf{S 2}$ & Medium & $\mathrm{Y}$ & & & $\mathrm{Y}$ & & \\
\hline S3 & Medium & & $\mathrm{Y}$ & & & & \\
\hline S4 & Large & & $\mathrm{Y}$ & $\mathrm{Y}$ & $\mathrm{Y}$ & & \\
\hline S5 & Large & $\mathrm{Y}$ & & $\mathrm{Y}$ & & & \\
\hline S6 & Medium & & & & $\mathrm{Y}$ & & \\
\hline S7 & Small & & $\mathrm{Y}$ & & & & \\
\hline S8 & Medium & & & & $\mathrm{Y}$ & & \\
\hline S9 & Medium & & $\mathrm{Y}$ & & & & \\
\hline S10 & Medium & & & $\mathrm{Y}$ & & & \\
\hline S11 & Medium & & & $\mathrm{Y}$ & & & \\
\hline S12 & Medium & $\mathrm{Y}$ & & & & & $\mathrm{Y}$ \\
\hline S13 & Medium & $\mathrm{Y}$ & & $\mathrm{Y}$ & & & \\
\hline S14 & Medium & $\mathrm{Y}$ & & $\mathrm{Y}$ & & $\mathrm{Y}$ & \\
\hline S15 & Medium & & & $\mathrm{Y}$ & & $\mathrm{Y}$ & \\
\hline S16 & Large & & & $\mathrm{Y}$ & & $\mathrm{Y}$ & \\
\hline S17 & Medium & & & Y & & & $\mathrm{Y}$ \\
\hline S18 & Medium & & & $\mathrm{Y}$ & & & \\
\hline S19 & Small & & $\mathrm{Y}$ & & & & \\
\hline $\mathbf{S 2 0}$ & Medium & & & $\mathrm{Y}$ & $\mathrm{Y}$ & & \\
\hline \multicolumn{2}{|c|}{ Model Size Summary } & \multicolumn{2}{|c|}{ Small: $3(15 \%)$} & Medium: & $(70 \%)$ & \multicolumn{2}{|c|}{ Large: $3(15 \%)$} \\
\hline \multicolumn{4}{|c|}{$\begin{array}{l}\text { - The time it takes to develop simulation model } \\
\text { - Number of variables } \\
\text { - Number of blocks }\end{array}$} & \multicolumn{4}{|c|}{$\begin{array}{l}\text { NOEAP }- \text { Number of elements or entities, activities, or } \\
\quad \text { process steps } \\
L O C \quad \text { - Lines of code } \\
A M I D \quad \text { - Amount of input data }\end{array}$} \\
\hline
\end{tabular}

The results show that there are no agreed metrics for simulation model size. One explanation might be that most of the simulation models developed by the participants are small or medium and for short-term use, therefore, they do not tend to pay attention to their model size. Moreover, S12 says that establishing metrics for simulation model size might be useful from an academic perspective; however, in a commercial environment the time it takes to develop a simulation model is more appropriate measure.

Exhibit II: Participants' views about model size.

S6: I guess number of blocks is one way to characterise the size of a model. And it was a couple of hundred blocks; I won't say it was a huge model. But it was relatively complicated by a medium size model. In terms of the number of the blocks in the model.

S8: Well I am consultant I measure in dollars...

S11: I would say if you are talking about elements rather than variables... if you got something like 10 to 15 elements you got a medium size model... less than 10 probably small... more than 20 means big... and what I mean by elements is machines, parts, buffers...

S12: I think it is natural for consultants (to measure in terms of time), because we would tend to when it comes to communicating with the client that how big the project is, and establishing its cost, we deal in the amount of time it takes to develop it... so yes we tend to talk in terms of development time... I think there is a fairly direct correlation between the amounts of time it takes and how difficult and how complex it is to develop it...

S14: ... so you are looking at 2 to 3 thousand servers within the simulation... and a workload of 10s of thousands of calls per day... so in terms or event list it is quite a big simulation problem...

\subsection{Complexity of simulation models}

The participants in this study have experience of developing simulation models of varying complexity. Complexity is perceived in a variety of ways by the participants and there seems 
to be no agreement that what could be a realistic measure for complexity. Table II summarizes participants' perceptions about the complexity of the models they develop.

Table II shows that 3 participants think that the models they develop are of low complexity; 12 participants, develop models of medium complexity; 5 participants develop highly complex models. This suggests that most of the participants mostly develop simulation models of low and/or medium complexity.

Most of the participants wanted to talk more about the complexity of the models rather than size, as quotes from S9 and S12 show in Exhibit III. Table II shows the complexity of models and a variety of possible measures of complexity as perceived by each participant. The number of interactions (NOI) between model elements, blocks or the variables is the most popular measure as perceived by the participants. They think that the greater the number of variables, blocks, elements or activities in the model, the more interactions will take place; therefore the model will be more complex. Those who had been involved in continuous simulation think that the higher the number of feedback loops $(N F B L)$, the higher the complexity of the model would be. S4 thinks that the number of questions $(N O Q)$ to be answered by the simulation study can be a measure of complexity because the greater the number of questions, greater will be the output values and analysis, hence making the simulation more complex. Some of the participants also believe that complexity in the data (CID) and complexity in the output (CIO) are good indications of the complexity of the simulation model. They think, therefore, this is directly related to the complexity of the simulation problem. S1 and S17 assume that the number of flows (NOF) in a simulation model can also be a measure for complexity.

Table II: Complexity of the models developed by the participants, and possible measures of complexity.

\begin{tabular}{|c|c|c|c|c|c|c|c|}
\hline Participants & Complexity & $\mathrm{NOI}$ & NOFBL & $N O Q$ & CID & CIO & $\mathrm{NOF}$ \\
\hline S1 & Low & $\mathrm{Y}$ & & & & & $\mathrm{Y}$ \\
\hline S2 & Medium & & $\mathrm{Y}$ & & & & \\
\hline S3 & High & & $\mathrm{Y}$ & & & & \\
\hline S4 & High & $\mathrm{Y}$ & & $\mathrm{Y}$ & & & \\
\hline S5 & Medium & & & & & & \\
\hline S6 & High & $\mathrm{Y}$ & & & & & \\
\hline S7 & Low & & & & & & \\
\hline S8 & Medium & $\mathrm{Y}$ & & & $\mathrm{Y}$ & $\mathrm{Y}$ & \\
\hline S9 & Medium & $\mathrm{Y}$ & $\mathrm{Y}$ & & & & \\
\hline S10 & Medium & $\mathrm{Y}$ & $\mathrm{Y}$ & & $\mathrm{Y}$ & $\mathrm{Y}$ & \\
\hline S11 & Medium & $\mathrm{Y}$ & & & & & \\
\hline S12 & Medium & $\mathrm{Y}$ & & & $\mathrm{Y}$ & $\mathrm{Y}$ & \\
\hline S13 & Medium & $\mathrm{Y}$ & $\mathrm{Y}$ & & & & \\
\hline S14 & Medium & $\mathrm{Y}$ & & & $\mathrm{Y}$ & & \\
\hline S15 & High & $\mathrm{Y}$ & & & & & \\
\hline S16 & High & $\mathrm{Y}$ & $\mathrm{Y}$ & & & & \\
\hline S17 & Medium & $\mathrm{Y}$ & & & $\mathrm{Y}$ & $\mathrm{Y}$ & $\mathrm{Y}$ \\
\hline S18 & Medium & $\mathrm{Y}$ & & & & $\mathrm{Y}$ & \\
\hline S19 & Low & & & & & $\mathrm{Y}$ & \\
\hline S20 & Medium & $\mathrm{Y}$ & & & & & \\
\hline \multicolumn{2}{|c|}{ Complexity } & \multicolumn{2}{|c|}{ Low: $3(15 \%)$} & \multicolumn{2}{|c|}{ Medium: $12(60 \%)$} & \multicolumn{2}{|c|}{ High: $5(25 \%)$} \\
\hline \multicolumn{4}{|c|}{$\begin{array}{l}\text { NOI } \quad \text { - Number of interactions } \\
N O F B L \text { - Number of feedback loops } \\
N O Q \quad \text { - Number of questions }\end{array}$} & \multicolumn{3}{|c|}{$\begin{array}{ll}\text { CID } & \text { - Complexity in data } \\
\text { CIO } & \text { - Complexity in the output } \\
\text { NOF } & \text { - Number of flows }\end{array}$} & \\
\hline
\end{tabular}

Most of the participants think that size and complexity are related most of the time; in general the larger the model size, the higher will be the complexity. Only S6 and S11 think that size and complexity are not necessarily related. S11 said that a model may be very big in 
terms of input data, number of blocks, and variables but different parts of a model may be replicating a similar structure, therefore, the model may not be as complex as it seems.

The results in this section show that most of the participants develop simulation models of low/medium complexity. Again there are no agreed metrics to measure the complexity of the models, and similarly no significant debate can be found in the literature about the complexity of models. However, the results show that complexity of models largely depends on the complexity of problems and size of the models.

Exhibit III: Participants' views about complexity.

S9: I think model complexity is probably more interesting (than model size)... but the point is that there is no agreed measure...

S2: I think there is a fairly direct correlation between the amount of time it takes and how difficult and how complex it is to develop it... it (size) isn't something that I find to be terribly important, what I find important is how long it takes...

S6: To me a model... the important thing about model is its degree of complexity... so complexity can be measured by the relationships amongst variables... my model probably had about 100 variables and it probably had 500 interrelationships amongst the variables... so that's where the complexity came in...

S12: (when asked about interrelationship between size and complexity) yes, in part... you can have a big model in size which is not very complex and other way round... but usually if you have bigger models they get more complex... because they tend to go in a network like structure.

S16 (relating complexity with time) Obviously not (the same thing)... but they are two sides of the same coin... adding complexity and adding size both take times...

\section{DISCUSSION}

These results provide a general picture the concepts of simulation model size and complexity held by simulation modellers. Size and complexity of models are important contextual factors; having an appreciation of simulation model size and complexity can be particularly helpful to adapt simulation practice according to the varying contexts.

The results show that there are no agreed metrics for simulation model size and no significant debate can be found about simulation model size in the literature. Simulation model size and complexity may have a relationship to the way people go about developing simulation models. Davis and Anderson [14] believe that simulation model size and complexity and the complexity of problem affect simulation model development, their maintenance, and documentation [15].

Brooks and Tobias [13] suggest that level of detail and complexity are the attributes that should be used to compare alternative models, however, they are very difficult to assess. One reason for it being difficult is that there are no agreed metrics available for simulation model size and complexity and little is found in the literature about it. Some of the measures suggested by our participants, such as, number of process steps, number of resources, number of products/projects, level of interaction between process steps, entities, and variables can be used to assess the level of detail of a model.

Brooks and Tobias [13] say that complexity is a good attribute to compare alternative models; they suggest that there are four commonly held concepts of complexity:

- computational complexity,

- structural complexity,

- behavioural complexity,

- logical and semantic complexity.

They suggest that graph theoretical approach, information theory approach, counting number of logical steps, and software engineering metrics for size and complexity can be used 
depending upon the type of model and simulation tool/language. A number of our participants, as shown in Table I and II, suggest that counting number of variables, parts, steps, block, entities, and lines of code can be used as a measure of size and complexity. Number of feedback loops $(N O F B L)$, number of interactions $(N O I)$ can be related with Brooks and Tobias's structural and behavioural complexity. Moreover, complexity in data (CID), and complexity in output (CIO) can be related with computational complexity. Since level of details and semantics of the model largely depends on the type of and number of questions to be answered, therefore, number of questions $(N O Q)$ can be related with semantic complexity.

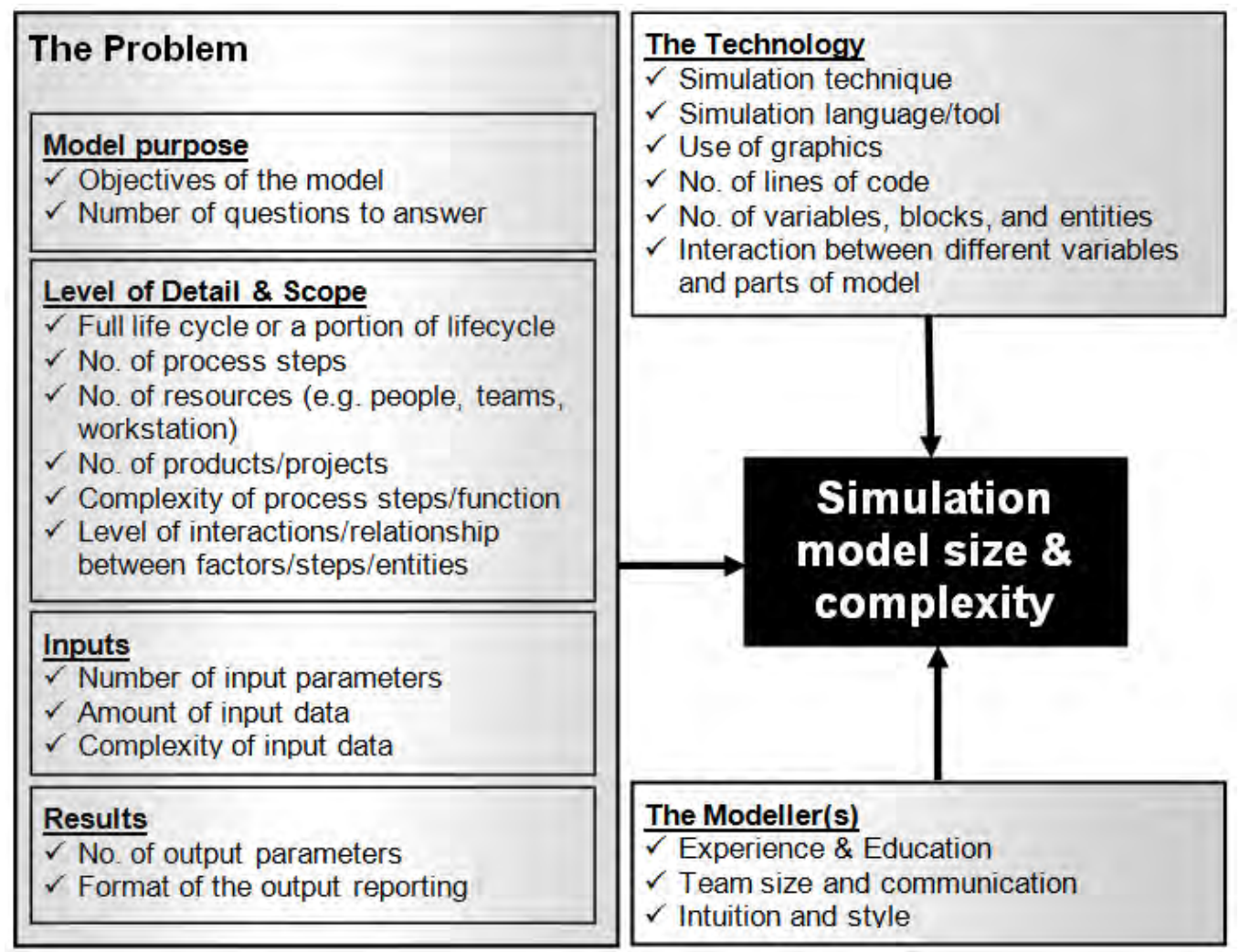

Figure 1: Origins of simulation model size and complexity.

Chwif et al. [2] identify several technical and non-technical reasons of simulation size and complexity. They believe that unclear objectives, lack of understanding of real system, inability to develop a good conceptual model, inability to translate conceptual model correctly to a computer model are some of the technical factors that increase a simulation model's complexity. While 'show off' factor (e.g. animation and sophisticated interface), 'include all' syndrome, and 'possibility' factor, including lot more than needed due to increased computing power are few non-technical reasons a model's complexity. They say that a complex model is eventually difficult to understand, difficult to validate, costly to run (in terms of time and computing resources), and takes more development time. Although our participants did not directly indicate any of non-technical reasons, increase in number of variables, entities, interactions etc. may be the direct result the non-technical factors.

We believe that understanding the origins and factors responsible for increasing size and complexity is as important as defining the concept of size and complexity. The data from this study and literature findings enable us identifying a number of factors and formulating a theoretical model showing the origins of size and complexity in simulation models. The simulation literature and data collected in this study suggests that complexity in simulation models can originate from a number of different sources and size of the model can be viewed 
from a number of different dimensions. Based on the collected data and literature review, we have developed a basic framework to understand the origins of size and complexity. The problem area, technology, and personal characteristics of modeller appear to be three main sources of model complexity and size (see Fig. 1).

The size and complexity of a model may be affected by the problem area and the nature of the problem. There are four main sources of size and complexity in a problem; purpose of the model, level of detail \& scope, inputs, and outputs. The purpose of the model i.e. objectives of the model and the number and type of questions to be answered can potentially affect simulation model size and complexity. More the number of questions to be answered, bigger and complex would be the model. Similarly, if the model's scope needs covering a lot of details of the problem in terms of life cycle portions, process steps, resources, and interactions between them, the model will be potentially bigger and more complex. Similarly, the number of input parameters (will increase with level of detail), the amount of input data, and complexity in collecting, understanding, translating/converting the input data will contribute towards increase complexity of a model and its size. Finally the number of output parameters (results) and how the output is to be processes, represented (e.g. in graphs, figures, tables etc.), understood, and explained can further affect a model's complexity and size.

Technology may also have an impact on a simulation model's size and complexity. A problem can be simulated through a number of different techniques and approaches or a hybrid approach might be required. Simulating a problem using discrete event approach might be more complex if the problem is more suitable for system dynamics approach. Similarly selection of appropriate simulation tool or programming language is another important area. Selection of wrong tool may introduce unnecessary complexity. Selection of simulation technique and tool depends both on the problem area and expertise and experience of a modeller. Number of lines of code, variables, or blocks in a simulation model may vary depending on tool and technique. Therefore it is important that an appropriate tool and technique be selected for simulation to avoid unnecessary complexity and size.

Finally, simulation model complexity also depends on individual characteristics of a modeller and his/her team members. More experienced the modeller, less complex the model will be and vice versa, as Chwif et al. [2] suggest that less experienced modellers fall to the 'include all' syndrome. Similarly experience also helps a modeller with appropriately determining a model objectives, questions, and scope and detail, and simulation tool and technique.

\section{CONCLUSION}

This research helps us understanding that what the size and complexity means to expert modellers and discover some possible origins of simulation model size and complexity. Understanding the origins and concepts of size and complexity may help modellers address the increasing size and complexity of simulation models and finish the simulation studies within budget as suggested by Jacobson and Yucesan [12]. The study identifies the problem area, technology, and modeller characteristics as the main sources of simulation model size and complexity and formulates a basic model of the origins of simulation model size and complexity.

This study falls short on exploring and discussing how client's interaction and intervention (though these translate into objectives and questions, output etc.), and commercial pressures of budget and cost may affect a simulation model's size and complexity. Although factors related to clients can be incorporated within problem area, the effect of client's interventions and interaction should also be studied in depth, since the client usually has a very dynamic role in a simulation study. 
This study only provides an overview of the concepts and origins of size and complexity held by some expert modellers. Like most qualitative studies, this study also has a limitation of small sample size; therefore, the results and interpretations cannot be generalized beyond the sample. Future in-depth studies should be conducted to further understand the phenomenon of simulation model size and complexity and quantitative studies might be needed to generalize the results to a larger sample of simulation modellers.

\section{REFERENCES}

[1] Henriksen, J. O. (2008). Taming the complexity dragon, Journal of Simulation, Vol. 2, No. 1, 317, doi:10.1057/palgrave.jos.4250029

[2] Chwif, L.; Barretto, M. R. P.; Paul, R. J. (2000). On simulation model complexity, Proceedings of the 2000 Winter Simulation Conference, Vol. 1, 449-455, doi:10.1109/WSC.2000.899751

[3] Balci, O.; Arthur, J. D.; Ormsby, W. F. (2011). Achieving reusability and composability with a simulation conceptual model, Journal of Simulation, Vol. 5, No. 3, 157-165, doi:10.1057/jos.2011.7

[4] Astrup, R.; Coates, K. D.; Hall, E. (2008). Finding the appropriate level of complexity for a simulation model: An example with a forest growth model, Forest Ecology and Management, Vol. 256, No. 10, 1659-1665, doi:10.1016/j.foreco.2008.07.016

[5] Arthur, J. D.; Sargent, R. G.; Dabney, J. B.; Law, A. M.; Morrison, J. D. (1999). Verification and validation: what impact should project size and complexity have on attendant V\&V activities and supporting infrastructure?, Proceedings of the 1999 Winter Simulation Conference, Vol. 1, 148155, doi:10.1109/WSC.1999.823064

[6] Ahmed, R.; Shah, M. (2015). Exploring the context and practices of expert simulation modellers, International Journal of Simulation Modelling, Vol. 14, No. 2, 265-277, doi:10.2507/ IJSIMM14(2)7.297

[7] Robinson, S. (2002). Modes of simulation practice: approaches to business and military simulation, Simulation Modelling Practice and Theory, Vol. 10, No. 8, 513-523, doi:10.1016/ $\underline{\text { S1569-190X(02)00117-X }}$

[8] Pidd, M. (1999). Just modeling through: A rough guide to modeling, Interfaces, Vol. 29, No. 2, 118-132, doi:10.1287/inte.29.2.118

[9] Powell, S. G. (1995). The teachers' forum: Six key modeling heuristics, Interfaces, Vol. 25, No. 4, 114-125, doi:10.1287/inte.25.4.114

[10] Ward, S. C. (1989). Arguments for constructively simple models, Journal of the Operational Research Society, Vol. 40, No. 2, 141-153, doi:10.1057/jors.1989.19

[11] Page, E. H.; Nicol, D. M.; Balci, O.; Fujimoto, R. M.; Fishwick, P. A.; L'Ecuyer, P.; Smith, R. (1999). Panel: Strategic directions in simulation research, Proceedings of the 1999 Winter Simulation Conference, Vol. 2, 1509-1520, doi:10.1145/324898.325314

[12] Jacobson, S. H.; Yücesan, E. (1999). On the complexity of verifying structural properties of discrete event simulation models, Operations Research, Vol. 47, No. 3, 476-481, doi:10.1287/opre.47.3.476

[13] Brooks, R. J.; Tobias, A. M. (1996). Choosing the best model: Level of detail, complexity, and model performance, Mathematical and Computer Modelling, Vol. 24, No. 4, 1-14, doi:10.1016/0895-7177(96)00103-3

[14] Davis, P. K.; Anderson, R. H. (2004). Improving the composability of DoD models and simulations, The Journal of Defense Modeling and Simulation: Applications, Methodology, Technology, Vol. 1, No. 1, 5-17, doi:10.1177/154851290400100101

[15] Sargent, R. G. (2013). Verification and validation of simulation models, Journal of Simulation, Vol. 7, No. 1, 12-24, doi:10.1057/jos.2012.20 